\title{
THE PROBLEM AND THE PROBLEMATIC: THE
}

\section{WORLD, ISLAM AND ISLAMIC WORLD}

\section{MUHAMMET S. KAFKASYALI}

Associate Professor, Department. of International Relations, Kirikkale University, Turkey

\begin{abstract}
This study explains the justification of the situation and even circumstances that must be described by the concept of the problem itself in order to decide what the likelihood of encountering those problems or circumstances are if the concept is purported with notions and equivalent concepts without knowing and understanding the concept.

First the definition, characteristics and elements of the concept of what a problem is manifested.

The theoretical basis of the difference between the problem and what is purported/considered/claimed to be a problem is explained.

The requirements for the correct definition and determination of the problem and the requirements for the elimination of the problem/problems from the perspective of the world, Islam and Islamic World are manifested. KEYWORDS: Problem, Islam, International System \& Islamic World
\end{abstract}

Received: Aug 09, 2017; Accepted: Aug 29, 2017; Published: Oct 21, 2017; Paper Id.: IJPSLIROCT20173

\section{INTRODUCTION}

The 'Problems of Islamic World' are the obstacles that prevent the protection of the integrity of the Islamic World on behalf of an understanding based on the acceptance of certain commonalities and its continuation through further solidification. The fact that there is an issue or problem is because the generated impact gives way to unwanted results. The issue/matter/problem is a reference to the unwanted results that will be generated by the consequences as well as the need to maintain the status/structure the deterioration of which is feared.

Problem and Islamic World

There are elements that must be evident in order to define the concept of "disruption in a functioning system" as a problem. There are four of these elements which are (i) time, (ii) man, (iii) process / system / mechanism and (iv) information/ news.

Time is the reason for the circulation of dispensing distinctions in terms of cause and effect, not only for the problem. Time is the opportunity to investigate what we call the reason and to understand the cause of any event. Due to its nature an event that happens later cannot be the cause of a previous event. As a result, the existence and the occurrence of an event in the cause-and-effect circle of a phenomenon, whether it carries the characteristics of the problem or not are possible with the presence of time and the opportunity that it provides. In other words, there is an element for each problem which is independent of the nature of the problem, its identification and assessments for its solution. 
We can ignore this element by saying that time is available and we cannot speak of the absence of time. However, being aware of the existence of time, the cause-effect relationship and the necessity generated by this relationship facilitates to understand the realm of existence of the problem as well as the limits of this domain.

The second element is the human element. There is a problem because there is a man. The source of the problem is man. For millions of years there was no problem in the world without human beings. The ensuing events did not cause a problem in the operation of the system. However, man is the only entity that can harm the functioning of the system and thus cause disruptions.

It can be thought that people already exist and for this reason this element can also be ignored. The reason for this is because there is a human element for every problem. However, being aware that the problem is a human condition and that the reason is human is extremely important in terms of determining and solving that problem.

The third element is the process / mechanism / system / structure element. In order to have a failure in a process, there has to be a process first. If there is a process that we can say is malfunctioning, we may study this malfunction in the process and resolve and eliminate it in order to maintain the processing. The first two elements are essential for a problem, however, they are not determining factors in the particular definition of the problem. In order to be a problem, there must be a structure that is functioning and a disruption that occurs in the functioning of this structure. In this case, it must be predictable, because the function is well-known. That is to say, it must be known how it happened/can happen, whether it can be remedied or not and how it can be remedied if it can be remedied. Otherwise the manifested situation should not be defined as a problem, but as an accident.

It is not really possible to be able to say that the structure which is called "Islamic World" and its problems which are mentioned and moreover, the correct diagnosis of these problems and even their resolution and elimination is a structure with processing. Of course, there are countries and geographies with Muslims or people who claim to be Muslims. However, there is no structure called the "Islamic World", which has a specific process / mechanism consisting of all the countries in which these people are located and which can predict the problems because its operation / mechanism is known. Therefore, we cannot talk about a problem or problems that can be called / identified as the problem / problems of the "Islamic World". Before the problem, its functioning must be determined and manifested whereas such a functioning does not exist.

Those who claim that the "Islamic World" has been correctly named and that its problems are the disruptions in the operation of the "Islamic World", and thus those who claim that there is a third element of the functioning, or there are those who assess erroneously what the problem and the problematic as well as their scope and elements which is the reason that we undertook this study. Therefore, let's evaluate the other elements as if there was a third element.

The fourth element is information / news. This last element is the element that has been examined since it is generally defined as a problem without examining the other elements. However, these examinations have been in the upper echelons in line with problem definitions done without concerns about defining the problem correctly and getting to the bottom of the problem. Moreover, the analyses in this echelon do not give the right result even at this level because the subject information has been handled differently.

The Information Element can be Assessed under Six Headings:

- $\quad$ System / mechanical knowledge 
- Knowing whether the system / mechanism is correct or not

- Know that there is a problem / be aware of the problem

- Know /be able to diagnose what the problem or malfunction is

- Know what the solution to the problem is

- Know how to reach the solution

Knowing the structure and the ability to determine the problems that may incur in this structure requires a solid knowledge regarding the components and the elements of the structure as well as the issues which are defined as problems. As a result, information will be the main element that guides us and dictates how we behave. When the subject is the Islamic World this information will enable the determination of the problems of Muslims as well as their solutions, it will include the characteristics of Muslims, be about Muslims as well as the characteristics which distinguish Muslims and information about the structure called Islamic World which is believed to have been structured and owned by Muslims.

The situation is the same in the upper echelons established by communities as it is on the scale of individuals and society comprised of individuals. Information will determine the constructed structure and whether this structure will be maintained or not as well as determine what the possible problems and their solutions are. Since the structure we have dealt with in this study is the Islamic World and the upper echelon of the Islamic World is comprised of the upper echelon of Muslims in more than one state (nation-states of course), first it will be necessary to assess the association of the states and information.

With the compulsory education system imposed by a nation-state, individuals and, ultimately, society is educated and become knowledgeable. The type of learning that people who are to be educated will be subjected to will be determined by the answer as to what is universally accepted and based on contemporary values. This answer will be the same for teaching as well. Either you will deliver an education configured on your own values and be outdated and reactionary, or you proceed in the direction of the rest of the world by delivering an education which is based on the acceptance/belief in right and wrong and be contemporary. As a result, you will be contemporary and your "con" will be the definer of the contemporary instead of the era/tempus (time).

People who were once educated/instructed according to rules based on their own culture and were educated/instructed according to these rules for hundreds of years are now being educated/instructed according to contemporary rulers. All aspects in the area of education / instruction will be determined according to contemporary rules from food and beverage customs to marriage procedures, from adult-minor relations to family relations, from the procedures of professional life with the determination of priorities in life. In fact, these contemporary rules and information will determine what the state needs to target and what must be done in line with these targets rather than what the individual and society should target.

Although the compulsory education-learning mandated by being a nation-state appears to provide the opportunity of adopting the values of the state and the nation, it ensures the instruction of knowledge with the rest of the world which has been generated in the single center which it is based on rather than its the values of the relevant state and nation. The consequences of delivering the same knowledge in harmony with the whole world are manifested as follows:

- Firstly the diagnosis of manifested problems as well as finding the solution for these problems will be based on 
this knowledge. However, the search for the source of the problem and its reason will be based on the acceptance and definitions of the mindset that generates this knowledge without being exposed to the scrutiny in terms of irregularity-regularity of the incumbent's own values. The applied knowledge will have been that generated by others.

- Over time, this will not only equalize the causes and solutions of the problems for every state and society, it will equalize the diversity of problems and the possible problems that could be manifested in all societies and states will now become the same.

- In the third stage when these possible problems and the reasons-results-solutions of these problems have become the same, the steps to be taken and generated and applied policies to prevent these problems will become the same. The same policies and planning for every society and state will become a requirement rather than a demand.

- Finally, the policies that are necessary at the outset and the application of these policies will be transformed into the desire and purpose of the society or state. Achieving a goal that is believed to be necessary will, of course, be desirable and an objective/goal.

The knowledge of the lower echelons which comprise the top echelon of the Islamic World needs to be of a quality tosustain this top echelon and resolve any problems which may be manifested in the light of this purpose as well as enable the prediction of whether the structural elements and bases of the work they primarily want to protect should be protected in their current state or whether modifications are required and to be able to predict what problems may be encountered and to take precautions to that effect. However, if the information to be defined before resolving the problem is not checked for correctness, an erroneous definition and diagnosis will result, which will make the solution impossible, and even culminate into a bottleneck.

\section{In Order to Eliminate Incorrect Knowledge it is Necessary to:}

- Teach and learn about information pertaining to the whole Islamic World, especially history for all relevant parties

- Realize that the information generated by others which is correct from their aspect is not correct for Islamic World

- Eliminate the barriers that prevent the world and Islamic World from knowing themselves as a result of the portrayal of characteristics of the Muslims differently and concealing the truth in many areas, especially history by other forces

- Correct intentionally modified information

- Re-evaluate compulsory and ideally presented processes such as civilization, modernization (westernization) and globalization from the perspective of Islamic World

- Believe that Islamic culture needs to be conserved in terms of fashion, popular culture (music, art, literature, cinema, etc.) within this framework and ensure that intense efforts are made in this regard.

\section{CONCLUSIONS}

There is an order which has a system and function, the order created by God. There is a process within this order 
that appears to deny it yet endeavors to surmount it, a system that has been established by man and has no order: the Modern International System. Islam is the essence of acting in a manner that does not cause disruption in the functioning of the system created by God which is natural and ethical. In fact, we cannot speak of a problem in the system because the Islamic World, which has been established by communities of people uniting around a joint issue is actually not a system with its functions and structure but is defined as a world, is not the name of a system. However, it is possible to talk about the functioning of the Modern International System, which has been established through the ignorance and impudence of man as an alternative to counter the order of God manifested by the essence of Islam and continues to generate oppression and problems.

The problem or problems are not the problems of the Islamic world. They are the problems of the Modern International System and therefore the problems of mankind. The only solution that will save mankind from the functioning of this present structure / system that constantly produces / manifest oppression is an intellectual flood. The mentality that will launch this field which will establish/ensure justice and which has been structured and established/created for mankind is the order that is based on Islam. 
